\title{
The role of biomarkers in the diagnosis and risk stratification of acute coronary syndrome
}

\author{
Sanoj Chacko*,1,2, Sohaib Haseeb², Benedict M Glover², David Wallbridge ${ }^{1}$ \& Alan Harper ${ }^{1}$ \\ ${ }^{1}$ KeeleCardiovascular Medicine, Keele University, Keele, Staffordshire, ST5 BG, UK \\ ${ }^{2}$ Heart Rhythm Service, Kingston General Hospital, Queen's University, Kingston, ON, Canada \\ * Author for correspondence: sanochacko@yahoo.co.uk
}

Coronary artery disease is a growing concern. Although traditional biomarkers, such as troponins and creatine kinase, play a central role in the diagnosis, risk stratification and management of coronary artery disease, they are unable to detect myocardial ischemia in the absence of necrosis. Therefore, early detection of ischemia in patients presenting with acute coronary syndrome still remains a burning question. High-sensitivity troponin is evolving as a reliable biomarker in this regard and has been absorbed into clinical practice. Biomarkers are currently the focus of immense interest as it not only helps with diagnosis and management but also helps to understand the pathophysiology of the disease process. In addition, analysis using a multimarker strategy has also proven to be a very useful tool in risk stratification. This review will focus on the biomarkers and its application in the diagnosis and risk stratification of acute coronary syndrome.

Lay abstract: Cardiovascular diseases are a common group of diseases that affect a large population and account for a significant number of deaths worldwide. Acute coronary syndrome (ACS) is a growing public health concern, where inadequate blood flow to the heart muscle results in reversible or irreversible damage to the cardiomyocyte. Early detection of ACS is crucial to deliver appropriate timely therapy to prevent myocardial necrosis and heart failure. Biomarkers are measurable and quantifiable biological substances that are used as indicators in the early diagnosis and management of patients with ACS. This article provides an overview of this topic and provides suggestions for the future.

First draft submitted: 14 March 2017; Accepted for publication: 11 September 2017; Published online: 27 October 2017

Keywords: acute coronary syndrome $\bullet$ biomarkers $\bullet$ creatine kinase $\bullet$ heart-type fatty acid-binding protein - myeloperoxidase $\bullet$ troponin

Cardiovascular disease is a very common diagnosis and a leading cause of death in both men and women. It accounts for $30 \%$ of deaths worldwide, including $40 \%$ in high-income countries and approximately $28 \%$ in the developing nations [1]. Data from the Framingham study, a 44-year follow-up of cohort, suggest that the incidence of coronary events significantly increases beyond the age of 65 , from 33 to $65 \%$ in men, and from 28 to $58 \%$ in women [2]. In 2007, the European Society of Cardiology, American College of Cardiology Foundation, American Heart Association and World Heart Federation task forces classified myocardial infarction (MI) as follows: Type $1=$ spontaneous $\mathrm{MI}$ as a result of primary coronary event such as plaque rupture; Type $2=\mathrm{MI}$ due to increased oxygen demand or reduced supply such as coronary spasm, embolism and arrhythmia; Type $3=$ sudden unexpected cardiac death secondary to presumed MI; Type $4=$ MI associated with percutaneous coronary intervention (PCI); and Type $5=\mathrm{MI}$ associated with coronary artery bypass surgery. Biomarkers play a key role in the diagnosis of acute coronary syndrome (ACS); however, it is a marker of myocardial necrosis. From the onset of ischemia, it could take as least as $20 \mathrm{~min}$ for myocardial necrosis to develop as previously demonstrated in the animal models, but for complete necrosis of all myocardial cells involving the culprit epicardial coronary vessel, it could take long as 2-4 h depending on other factors such as presence of collaterals, persistent or intermittent coronary occlusion and ischemia preconditioning [3]. The diagnosis of ACS has traditionally depended upon a combination of ischemic symptoms, ECG changes and elevation in serum biomarkers. However, the symptoms are often rather atypical 


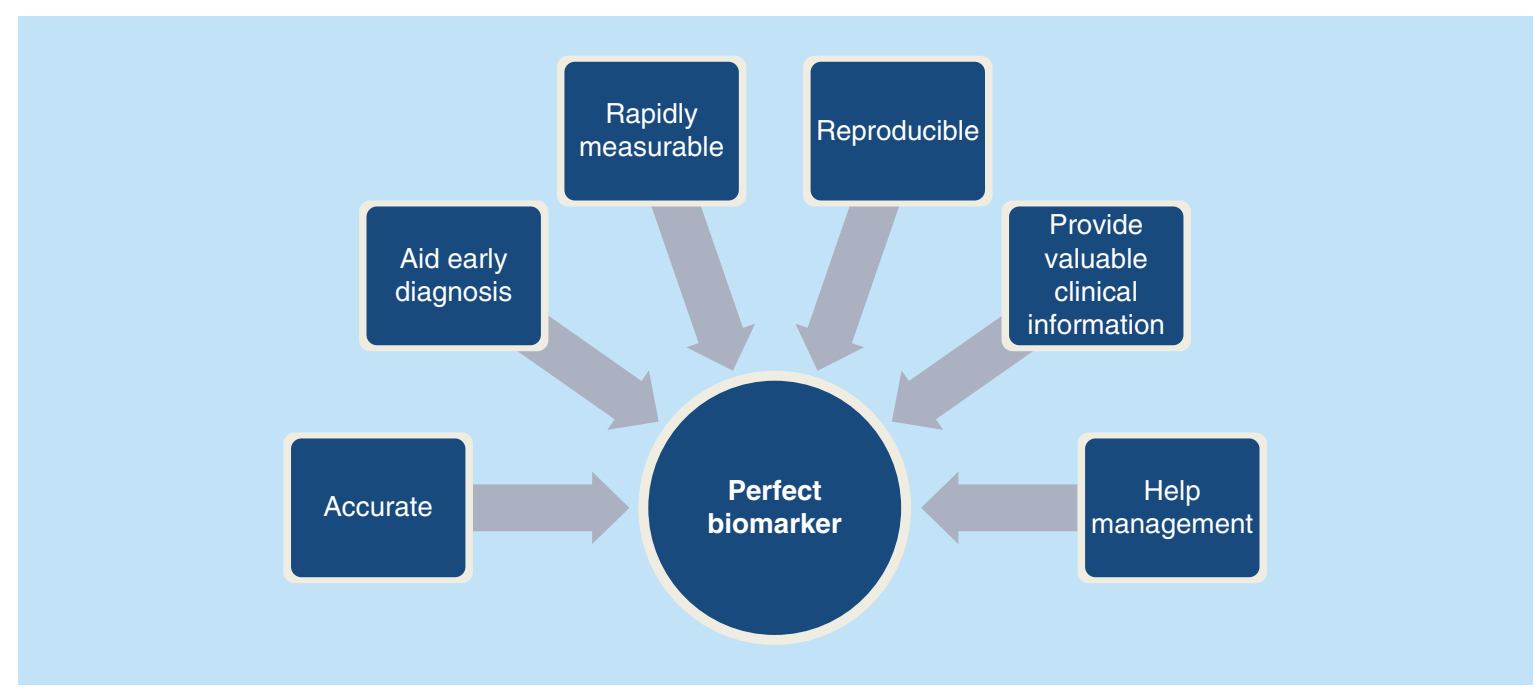

Figure 1. Represents the benchmarks of a perfect biomarker.

or absent, and around 33\% of the patients arriving at the emergency department with MI may not have chest pains [4]. Similarly, ECG changes that aid with early diagnosis may be nonspecific or even absent in around 40\% of the patients [5]. Moreover, ST-segment changes are also observed in other cardiac conditions like pericarditis, left ventricular hypertrophy, cardiomyopathies and channelopathies, which can add to the diagnostic dilemma.

Despite improved laboratory assays for cardiac-specific biomarkers and a revised definition for ACS, early detection of coronary ischemia in unselected patients with chest pain remains a major challenge [6]. Although conventional biomarkers play a significant role in diagnosing ACS, it is still insufficient in picking up those highrisk patients in the early phase of myocardial ischemia, which results in a delayed treatment plan [7]. Thus, growing interest in the identification of novel biomarkers of myocardial ischemia has spiked in recent years.

\section{Biomarkers}

Biomarker, a term introduced in 1989 as a measurable and quantifiable biological substance used as an indicator of a specific biologic state relevant to a specific disease process [8]. In 2001, the 'Biomarker Definitions Working Group' standardized the definition of a biomarker "a characteristic that is objectively measured and evaluated as an indicator of normal biologic process, pathogenic process or pharmacologic responses to therapeutic intervention" [9]. Biomarkers play an important and indisputable role in the diagnosis and management of patients with ACS. They also provide information regarding the pathophysiology, and are useful in improving treatment strategies for patient care. Specific biomarkers reflect different components of the pathophysiology of ACS: troponins are markers for myocardial necrosis, C-reactive protein and myeloperoxidase (MPO) reflect inflammatory process, and natriuretic peptides indicate neurohormonal activation and hemodynamic stress. The emergence of cardiac biomarkers has not only provided a unique insight into the disease process, but also represent an essential criterion in the definition of ACS [10].

There have been considerable advances over the years that have led to a comprehensive understanding of the pathophysiology and bimolecular basis of coronary artery disease (CAD). As a consequence, novel cardiac biomarkers are an exciting and fascinating area of research. Novel technologies have allowed us to screen large samples of blood in a much reduced time than ever before. This provides a larger scope for researchers to apply their understanding according to the ever-growing clinical demand. There has been rapid growth in the number of novel biomarkers that emphasizes the importance of their evaluation [11]. An ideal biomarker should convincingly demonstrate its value and utility in helping with diagnosis, disease stratification and prognostication, beyond that of the existing markers. Benchmarks of a perfect biomarker and the fundamental principles of a disease-specific biomarker are shown in Figures $1 \& 2$, respectively. So far, the largest wealth of knowledge lies with troponins, MB isoenzyme of creatine kinase (CK-MB), myoglobin and lactate dehydrogenase. However, many other potential biomarkers have emerged and are under intense research. 


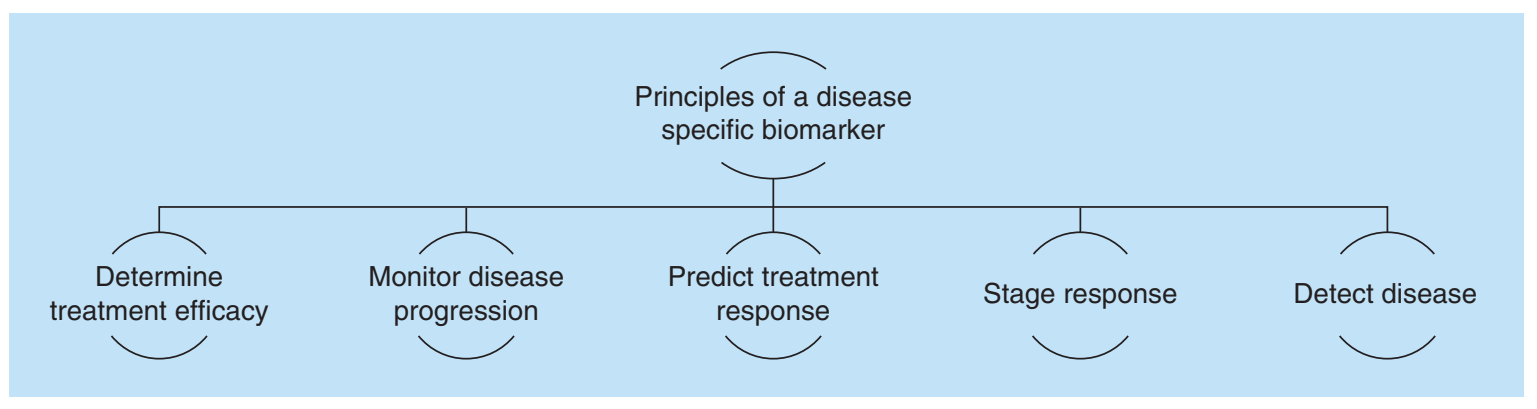

Figure 2. The fundamental principles of a disease-specific biomarker.

\begin{tabular}{|llll|}
\hline \multicolumn{4}{|l}{ Table 1. Time course of biomarker response in myocardial infarction. } \\
\hline Markers & Onset & Peak & Duration \\
\hline Troponin & $3-12 \mathrm{~h}$ & $18-24 \mathrm{~h}$ & 10 days \\
\hline Creatine kinase (total and MB) & $3-12 \mathrm{~h}$ & $18-24 \mathrm{~h}$ & $36-48 \mathrm{~h}$ \\
\hline Lactate dehydrogenase & $6-12 \mathrm{~h}$ & $24-48 \mathrm{~h}$ & $6-8$ days \\
\hline Myoglobin & $1-4 \mathrm{~h}$ & $6-7 \mathrm{~h}$ & $24 \mathrm{~h}$ \\
\hline
\end{tabular}

\section{Biomarkers in the diagnosis \& risk stratification of ACS}

ACS, a broad term used in clinical practice, represents three primary presentations that form a part of the continuum of ACS. Depending upon patients' cardiac symptoms, ECG changes and biomarker response, the definition is classified as: ST elevation myocardial infarction: an ST-segment elevation in the ECG with typical cardiac chest pain and rise in cardiac enzymes; non-ST elevation myocardial infarction (NSTEMI): presentation of cardiac chest pain with raised cardiac enzymes in the absence of an ST-segment elevation, but may have either ST depression, T inversion or equivocal changes; unstable angina (UA): presentation of cardiac chest pain, at rest, in the absence of any enzymatic rise, but a high risk of developing a future heart attack. Cardiac biomarkers play a major role in the diagnosis of ACS, particularly in situations where other diagnostic evidences are lacking, such as atypical cardiac chest pain or nonspecific ECG changes. Several biomarkers have emerged as a useful diagnostic tool in ACS, such as troponin, CK, CK-MB, LDH and myoglobin. By definition, elevation of one or more of the above markers is seen in all ACS patients [12], at various time courses (Table 1). However, because of their increased specificity compared with other markers, serum troponins are currently the preferred biomarker for the diagnosis of a myocardial injury.

\section{Troponins}

Cardiac troponins $\mathrm{I}$ and $\mathrm{T}$ are proteins that control the calcium-mediated interaction of actin and myosin. Troponins are currently the golden standard test for the diagnosis of ACS. They are elevated at 3-6 h after the onset of symptoms, and remain elevated for up to 10 days. This not only helps with diagnostic precision, but also permits late diagnosis [13]. According to the European Society of Cardiology and American College of Cardiology consensus recommendations, troponin concentration above 99th percentile of normal is considered the diagnostic criteria for MI [14,15] and most studies support this diagnostic criterion [16]. Troponin has a good specificity and sensitivity for detecting myocardial necrosis and has also emerged as a powerful predictor of prognosis [17]. The correlation of the degree of troponin elevation with mortality has been shown in major trials like the Thrombolysis in Myocardial Infarction IIIB trial [18], in which troponin levels were measured at baseline in 1404 patients with UA or NSTEMI. The authors of this trial reported that the degree of troponin rise correlated with 42-day mortality, and moreover, a rise in troponin levels correlated with a progressive increase in risk of mortality. Similar findings were also noted in the Global Utilization of Streptokinase and t-PA for Occluded Coronary Arteries [19]. The Fragmin During Instability in Coronary-Artery Disease trial studied 917 patients with NSTEMI and showed that elevated troponin, measured within $24 \mathrm{~h}$ of presentation was associated with an increased incidence of death at a mean follow-up of 37 months [13,20].

Cardiac troponins can play an important role in primary prevention for cardiovascular event. The BiomarCaRE consortium by Blankenberg et al. was an investigation undertaken to evaluate troponin I concentration distributions 
across the content of Europe for risk stratification of cardiovascular and noncardiovascular mortality. The study cohort consisted of ten population-based studies, which encompassed 74,738 subjects with measurements for troponin I concentrations. Investigators noted that with troponin concentrations above $6 \mathrm{ng} / \mathrm{l}$, an improved risk prediction was observed with a hazard ratio of 1.87 (95\% CI: 1.72-2.03; $\mathrm{p}<0.001)$ for cases, and a hazard ratio of 0.013 (95\% CI: 0.011-0.016) for noncases. Troponin I concentrations in healthy subjects were associated with fatal cardiovascular events, overall mortality and cardiovascular disease. After an extensive analysis of studies, the authors concluded that an improvement of prediction of risk for cardiovascular death and overall mortality occurs with the addition of troponin I concentrations [21].

In addition to its diagnostic and prognostic usefulness, troponins are also used as a guide for selecting patients who may benefit from specific treatment strategies, including but not limited to low molecular weight heparin and cardiac catheterization. Despite troponin's obvious strengths and its significance in the management of ACS, it has some limitations: a concerning issue is that troponin concentration above the 99th percentile to the normal is used as the cut-off point for the diagnosis of MI; 99th percentile of troponin T is about $0.01 \mu \mathrm{g} / \mathrm{l}$, while most routine laboratory assays do not reliably measure levels below $0.03 \mu \mathrm{g} / \mathrm{l}$. Therefore, patients with troponin concentration between 0.01 and $0.03 \mu \mathrm{g} / \mathrm{l}$, who will currently be classified as possible ACS according to the present criteria, are potentially missed and categorized as low risk [22]. Another point in question is the analytical techniques that involve immunoassays as opposed to the enzymatic methods used for creatine kinase. There are variations in the sensitivity and specificity of various immunoassays due to the lack of standardization and variations in antibody cross-reactivities to the various detectable forms of troponins [23,24]. Troponins are elevated in the range of 3$12 \mathrm{~h}$ after a myocardial injury, and detectable concentration is an indication of an irreversible myocardial injury, suggesting a delay relative to the onset of ischemia [25]. Troponins are unable to detect myocardial ischemia in the absence of necrosis, and hence unable to yield early diagnosis. Although troponin provides improved specificity for the detection of myocardial injury, its elevation does not necessarily imply the onset of ACS. Troponin elevation occurs in a variety of other clinical conditions including a moderate-to-severe pulmonary embolism with an acute right heart overload, heart failure and myopericarditis [26]. However, troponin elevations are usually modest in these disorders when contrasted to the more prolonged elevation that occurs with acute myocardial injury [27]. The risk assessment of cardiac biomarkers in patients with renal failure and normal renal function is not equivalent; therefore, the significance of troponin elevation in the setting of end-stage renal disease is controversial [28]. Although several studies in the past have reported that troponin rise in patients with chronic renal failure is an independent predictor of adverse outcome, it is possible to have an elevated troponin in the absence of true cardiac injury, due to possible re-expression of cardiac troponin in uremic myopathic skeletal muscles and due to reduced clearance.

\section{Higher sensitivity troponin}

The older assays are referred to as 'conventional' and the newer assays as 'high sensitivity'. As the name stands, the older assays are less sensitive than the newer ones. According to the 2012 expert consensus, to be classified as a high-sensitivity assay, it should have a coefficient of variance of $<10 \%$ at the 99th percentile value, and a concentration below the 99th percentile should be detectable above the assay's limit of detection for $>50 \%$ of healthy individuals in the population of interest [29]. High-sensitivity troponin assays detect concentrations that are tenfold lower than those detected with the standard assay, and these have shown to be independently associated with adverse cardiac outcome in patients with heart failure and stable CAD [30,31]. In a community-based prospective study of 1499 individuals, Xiao et al. showed that detectable troponin levels were noted in 820 participants of which most of them were older male with history of hypertension, diabetes, impaired renal function and was associated with increased subsequent risk of all-cause mortality and cardiovascular events [32]. A recent study further evaluated the association between high-sensitive troponin and 5-year outcome among patients with diabetes mellitus and stable CAD. The study showed a strong and consistent association between baseline concentrations of circulating troponins and the risk of all-cause mortality, ACS, stroke and heart failure [33]. These studies suggest that, in addition to the diagnostic role of troponin as a marker of ACS, it can also assess ongoing myocardial injury in stable patients and even seemingly healthy populations and could be employed as a reliable tool for risk stratification. Troponin's improved sensitivity has allowed us to detect its first low-level elevations, which are detectable within $90-180 \mathrm{~min}$ of an indexed cardiac event [34,35].

Despite the increase in accuracy and early detection of ACS with a high-sensitivity assay, there are still a few concerns. Research has shown a nominal level of high-sensitivity troponin at baseline in apparently healthy subjects, and the presence of biological variability over time. These changes could be due to circadian rhythm, 
seasonal changes or a random biological fluctuation around an inherent set point, specific to an individual $[4,36]$. Due to the variability with time and detectable levels in a healthy state, further investigations are needed to apply this in a more reliable manner.

As the sensitivity and precision of troponin assay improves, false positives have become less of a problem. However, given that there is a large differential diagnosis generated by positive troponins, positive results should be closely correlated with clinical presentations. Although troponin is a strong prognostic marker for cardiovascular death and recurrent ischemic events, further investigations are needed to provide thresholds to guide therapeutic strategies. With recent advances in technology, high-sensitivity troponins have emerged as an important biomarker with an added advantage in early diagnosis and risk stratification for patients with ACS.

\section{Creatine kinase}

The CK-MB was the preferred marker of cardiac injury for several years. It has a high specificity for cardiac tissue, particularly in patients with ischemic symptoms in the absence of skeletal muscle damage. Increases in CK usually begin $4-6 \mathrm{~h}$ after the onset of MI, and peaks at around 18-24 h, while returning to baseline levels by 36-48 h [37]. A total CK elevation of twofold above normal, with a simultaneously elevated CK-MB is required for the diagnosis of an MI. However, the important drawback of this is a false positive result, which can be due to skeletal muscle damage from trauma, cardiopulmonary resuscitation, defibrillation, cardiac and noncardiac surgical procedures, cocaine abuse, intramuscular injections, convulsions, alcohol intoxication, hypothyroidism, renal failure and pulmonary embolism [38,39]. In the diagnosis of ACS, CK is comparatively less sensitive and specific than troponin. Several trials in the past have compared troponin with CK-MB. A review of around 30,000 patients in the multicenter, 'Can Rapid Risk Stratification of Unstable Angina Patients Suppress Adverse Outcomes with Early Implementation of the American College of Cardiology (ACC)/Americal Heart Association (AHA) (CRUSADE)' trial, showed that troponin was more sensitive and has an enhanced prognostic value than CK-MB [40]. Similarly, in a report of over 10,000 patients with ACS from the multicenter GRACE registry showed that in-hospital mortality was highest when both troponin and CK-MB were positive (7.7\%), intermediate in troponin-positive/CK-MB-negative patients (3.9\%) and lowest in patients in whom both markers were negative, and those who were troponin-negative/CK-MB-positive (1.7 and 2.3\%, respectively) [41]. The CARMAGUE study assessed European (EU) and North American (NA) guidelines for cardiac biomarkers using a web-based questionnaire. Compilation of 533 hospitals (422 EU institutions; $91 \mathrm{NA}$ institutions) revealed that cardiac troponins were the preferred biomarkers (99.5 EU; $98.7 \mathrm{NA}$ ) with the majority of healthcare institutions using it as a first-line biomarker. Differing laboratory practices and biomarker availability lead to differences in the use of cardiac biomarkers [42], but with the evidence from this study, it could be argued that creatine kinase, LDH and myoglobin are less important than cardiac troponins.

\section{Heart-type fatty acid-binding protein}

Heart-type fatty acid-binding protein (H-FABP) is a small cytosolic protein that is concentrated in the cardiomyocyte. It transports fatty acids in the myocytes and is released in the circulation in response to ischemic insult. Several studies have shown that H-FABP is a sensitive marker of ACS [43]. Seino et al. measured H-FABP levels in 371 consecutive patients with acute chest pain and suspected MI [44]. There were 68 patients who presented within $2 \mathrm{~h}$ of symptoms. The sensitivity of MI at $2 \mathrm{~h}$ was significantly higher with serum H-FABP compared with cardiac troponin T or myoglobin (89 vs 22 and 38\%, respectively). However, cardiac troponin T had a significantly higher specificity (94 vs 52\%). H-FABP has shown to provide valuable information in the multimarker approach in ACS, and when measured in combination with troponins and BNP, H-FABP has proven to be very useful in identifying patients who are at an increased risk of a worsening heart failure, death and rehospitalization. Moreover, it has also shown to be useful in estimating the size of the infarct. H-FABP appeared to provide incremental information beyond the established biomarkers; however, this has not been compared and established [45], and there are no studies which directly compare H-FABP with troponins, although its smaller size, rapid release and clearance offer some theoretical advantage over troponins [46]. H-FABP appears to be a very promising biomarker of myocardial injury; however, more studies are required to support these preliminary findings.

\section{Myeloperoxidase}

MPO is a hemoprotein mainly released by activated neutrophils, monocytes and tissue macrophages. It is emerging as a new biomarker of inflammation and has been proposed as a useful diagnostic tool in patients admitted with 
ACS [47]. MPO appears to play an important role in atherogenesis by its involvement in low-density lipoprotein oxidation. Recent studies have suggested an association between plasma levels of MPO and coronary atherosclerosis, and this enzyme is also found to be abundant in ruptured plaques [48]. MPO is also emerging as a useful tool in primary prevention to assess the risk for future coronary event; however, only few important studies have examined the role of MPO as a marker of risk for CAD. In relation to this, Zhang et al. showed that the serum MPO activity was higher in patients with CAD when compared with angiographically verified normal subjects [49]. MPO has also been very useful in the setting of ACS where high-circulating MPO levels were detected in patients with UA and acute MI when compared with patients with stable CAD, suggesting that MPO levels are a marker of instability. Its potential usefulness in risk stratification in ACS was examined in the CAPTURE trial [50]. CAPTURE trial enrolled 1265 patients with ACS. At baseline, MPO was detectable in the serum samples of all subjects, with a median of $287 \mu \mathrm{g} / \mathrm{l}$. However, when compared with the low levels $(<222 \mu \mathrm{g} / \mathrm{l})$, MPO levels of $>350 \mu \mathrm{g} / \mathrm{l}$ was associated with significant event rates at $72 \mathrm{~h}, 30$ days and 6 months' follow-up. There are also studies looking at MPO's long-term outcome in patients with ACS, Mocatta et al. studied 512 patients with acute MI and found a significant association of raised MPO levels and repeat cardiac events [51]. MPO is a marker of inflammation and oxidative stress that is emerging as a potential biomarker of ACS; however, there are only few strong quantitative analyses available; therefore, more studies are required to determine the precise role of MPO in ACS.

\section{Conclusion \& future perspective}

The traditional biomarkers currently available, such as troponins and CK have played a major role in the diagnosis, risk stratification and management of ACS. Extensive research has led to the discovery and development of potential novel biomarkers such as MPO, H-FABP and are under intense validation and research. With the emergence of novel markers, complimentary analysis using a multimarker strategy has also proven to be a very useful tool in risk stratification.

The number of novel biomarkers are expected to grow over the years to come, with the ultimate goal being the identification of markers which could potentially add more value and provide additional information to improve patient care. With the omics approach growing immensely, clinicians and researchers can use metabolomics to study these processes at the molecular level, further aiding in optimized therapies to be translated to clinical practice.

\section{Executive summary}

- Biomarkers are a characteristic that is objectively measured and evaluated as an indicator of normal biologic process, pathogenic process or pharmacologic responses to therapeutic intervention.

- Acute coronary syndrome (ACS) is a broad term used in clinical practice, representing three primary presentations that form a part of the continuum of ACS (ST elevation myocardial infarction, non-ST elevation myocardial infarction, angina).

- Over the years, many biomarkers have emerged as a useful diagnostic tool in ACS, most importantly troponins, and to a lesser degree CK, MB isoenzyme of creatine kinase.

- High-sensitivity troponins are currently the gold standard test for the diagnosis and management of ACS.

- Novel biomarkers are emerging as an area of immense interest in the diagnosis and risk stratification of ACS.

- Complimentary analysis using multibiomarker strategy has also proven to be a useful risk stratification tool.

Financial \& competing interests disclosure

The authors have no relevant affiliations or financial involvement with any organization or entity with a financial interest in or financial conflict with the subject matter or materials discussed in the manuscript. This includes employment, consultancies, honoraria, stock ownership or options, expert testimony, grants or patents received or pending, or royalties.

No writing assistance was utilized in the production of this manuscript.

Open access

This work is licensed under the Creative Commons Attribution 4.0 License. To view a copy of this license, visit http:// creativecommons.org/licenses/by/4.0/

\section{References}

Papers of special note have been highlighted as: $\bullet$ of interest; $\bullet \bullet$ of considerable interest 
1 Lopez AD, Mathers CD, Ezzati M, Jamison DT, Murray CJ. Global and regional burden of disease and risk factors, 2001: systematic analysis of population health data. Lancet 367(9524), 1747-1757 (2006).

- Trends about incidence of mortality, prevalence and estimates of the global burden of disease.

2 Lerner DJ, Kannel WB. Patterns of coronary heart disease morbidity and mortality in the sexes: a 26-year follow-up of the Framingham population. Am. Heart J. 111(2), 383-390 (1986).

3 Thygesen K, Alpert JS, White HD, Joint ESC/ACCF/AHA/WHF Task Force for the Redefinition of Myocardial Infarction. Universal definition of myocardial infarction. Eur. Heart J. 28(20), 2525-2538 (2007).

- Expert consensus document on myocardial infarction's (MI) universal definition.

4 Canto JG, Shlipak MG, Rogers WJ et al. Prevalence, clinical characteristics, and mortality among patients with myocardial infarction presenting without chest pain. JAMA 283(24), 3223-3229 (2000).

5 Rouan GW, Lee TH, Cook EF, Brand DA, Weisberg MC, Goldman L. Clinical characteristics and outcome of acute myocardial infarction in patients with initially normal or nonspecific electrocardiograms (a report from the Multicenter Chest Pain Study). Am. J. Cardiol. 64(18), 1087-1092 (1989).

6 Eggers KM, Oldgren J, Nordenskjöld A, Lindahl B. Diagnostic value of serial measurement of cardiac markers in patients with chest pain: limited value of adding myoglobin to troponin I for exclusion of myocardial infarction. Am. Heart J. 148(4), 574-581 (2004).

7 Zimmerman J, Fromm R, Meyer D et al. Diagnostic marker cooperative study for the diagnosis of myocardial infarction. Circulation 99(13), 1671-1677 (1999).

8 Molitoris BA, Melnikov VY, Okusa MD, Himmelfarb J. Technology Insight: biomarker development in acute kidney injury - what can we anticipate? Nat. Clin. Pract. Nephrol. 4(3), 154-165 (2008).

9 Biomarkers Definitions Working Group. Biomarkers and surrogate endpoints: preferred definitions and conceptual framework. Clin. Pharmacol. Ther. 69(3), 89-95 (2001).

10 Howie-Esquivel J, White M. Biomarkers in acute cardiovascular disease. J. Cardiovasc. Nurs. 23(2), 124-131 (2008).

11 O’Donoghue M, Morrow DA. The future of biomarkers in the management of patients with acute coronary syndromes. Curr. Opin. Cardiol. 23(4), 309-314 (2008).

12 Lindahl B, Venge P, Wallentin L. Relation between troponin $\mathrm{T}$ and the risk of subsequent cardiac events in unstable coronary artery disease. The FRISC study group. Circulation 93(9), 1651-1657 (1996).

13 Lindahl B, Toss H, Siegbahn A, Venge P, Wallentin L. Markers of myocardial damage and inflammation in relation to long-term mortality in unstable coronary artery disease. FRISC Study Group. Fragmin during Instability in Coronary Artery Disease. N. Engl. J. Med. 343(16), 1139-1147 (2000).

14 Lindahl B, Venge P, Wallentin L. Troponin T identifies patients with unstable coronary artery disease who benefit from long-term antithrombotic protection. Fragmin in Unstable Coronary Artery Disease (FRISC) Study Group. J. Am. Coll. Cardiol. 29(1), 43-48 (1997).

15 Jneid H, Alam M, Virani SS, Bozkurt B. Redefining myocardial infarction: what is new in the ESC/ACCF/AHA/WHF third universal definition of myocardial infarction? Methodist DeBakey Cardiovasc. J. 9(3), 169-172 (2013).

- New universal definition of MI.

16 Morrow DA, Cannon CP, Rifai N et al. Ability of minor elevations of troponins I and T to predict benefit from an early invasive strategy in patients with unstable angina and non-ST elevation myocardial infarction: results from a randomized trial. JAMA 286(19), 2405-2412 (2001).

17 Hamm CW, Goldmann BU, Heeschen C, Kreymann G, Berger J, Meinertz T. Emergency room triage of patients with acute chest pain by means of rapid testing for cardiac troponin T or troponin I. N. Engl. J. Med. 337(23), 1648-1653 (1997).

18 Sabatine MS, Morrow DA, Giugliano RP et al. Implications of upstream glycoprotein IIb/IIIa inhibition and coronary artery stenting in the invasive management of unstable angina/non-ST-elevation myocardial infarction: a comparison of the Thrombolysis In Myocardial Infarction (TIMI) IIIB trial and the Treat angina with Aggrastat and determine Cost of Therapy with Invasive or Conservative Strategy (TACTICS)-TIMI 18 trial. Circulation 109(7), 874-880 (2004).

- Thrombolysis in Myocardial Infarction IIIB trial evaluating the correlation of the degree of troponin elevation with mortality, MI and rehospitalization outcomes.

19 Ohman EM, Armstrong PW, Christenson RH et al. Cardiac troponin T levels for risk stratification in acute myocardial ischemia. GUSTO IIA Investigators. N. Engl. J. Med. 335(18), 1333-1341 (1996).

20 Wallentin L, Ohlsson J, Swahn E. Low-molecular-weight heparin during instability in coronary artery disease. Lancet 347(9001), 561-568 (1996).

21 Blankenberg S, Salomaa V, Makarova N et al. Troponin I and cardiovascular risk prediction in the general population: the BiomarCaRE consortium. Eur. Heart J. 37(30), 2428-2437 (2016).

-. BiomarCaRE consortium study evaluating troponin I concentration distributions across Europe for risk stratification of cardiovascular and noncardiovascular mortality. 
22 Apple FS, Quist HE, Doyle PJ, Otto AP, Murakami MM. Plasma 99th percentile reference limits for cardiac troponin and creatine kinase MB mass for use with European Society of Cardiology/American College of Cardiology consensus recommendations. Clin. Chem. 49(8), 1331-1336 (2003).

23 Shi Q, Ling M, Zhang X et al. Degradation of cardiac troponin I in serum complicates comparisons of cardiac troponin I assays. Clin. Chem. 45(7), 1018-1025 (1999).

24 Adams JE, Abendschein DR, Jaffe AS. Biochemical markers of myocardial injury. Is MB creatine kinase the choice for the 1990s? Circulation 88(2), 750-763 (1993).

25 Puleo PR, Guadagno PA, Roberts R et al. Early diagnosis of acute myocardial infarction based on assay for subforms of creatine kinase-MB. Circulation 82(3), 759-764 (1990).

26 Korff S, Katus HA, Giannitsis E. Differential diagnosis of elevated troponins. Heart Br. Card. Soc. 92(7), 987-993 (2006).

27 Apple FS. Tissue specificity of cardiac troponin I, cardiac troponin T and creatine kinase-MB. Clin. Chim. Acta 284(2), 151-159 (1999).

28 Collinson PO, Hadcocks L, Foo Y et al. Cardiac troponins in patients with renal dysfunction. Ann. Clin. Biochem. 35(3), 380-386 (1998).

29 Apple FS, Collinson PO, IFCC Task Force on Clinical Applications of Cardiac Biomarkers. Analytical characteristics of high-sensitivity cardiac troponin assays. Clin. Chem. 58(1), 54-61 (2012).

30 Masson S, Anand I, Favero C et al. Serial measurement of cardiac troponin T using a highly sensitive assay in patients with chronic heart failure: data from two large randomized clinical trials. Circulation 136(8), 280-288 (2011).

31 Beatty AL, Ku IA, Christenson RH, DeFilippi CR, Schiller NB, Whooley MA. High-sensitivity cardiac troponin T levels and secondary events in outpatients with coronary heart disease from the heart and soul study. JAMA Intern. Med. 173(9), 763-769 (2013).

32 Xiao W, Cao R, Liu Y et al. Association of high-sensitivity cardiac troponin T with mortality and cardiovascular events in a community-based prospective study in Beijing. BMJ Open 7(6), e013431 (2017).

33 Everett BM, Brooks MM, Vlachos HEA, Chaitman BR, Frye RL, Bhatt DL. Troponin and cardiac events in stable ischemic heart disease and diabetes. N. Engl. J. Med. 373(7), 610-620 (2015).

34 Yusuf S, Reddy S, Ounpuu S, Anand S. Global burden of cardiovascular diseases: part II: variations in cardiovascular disease by specific ethnic groups and geographic regions and prevention strategies. Circulation 104(23), 2855-2864 (2001).

35 Okrainec K, Banerjee DK, Eisenberg MJ. Coronary artery disease in the developing world. Am. Heart J. 148(1), 7-15 (2004).

36 Alpert JS, Thygesen K, Antman E, Bassand JP. Myocardial infarction redefined - a consensus document of The Joint European Society of Cardiology/American College of Cardiology Committee for the redefinition of myocardial infarction. J. Am. Coll. Cardiol. 36(3), 959-969 (2000).

37 Newby LK, Roe MT, Chen AY et al. Frequency and clinical implications of discordant creatine kinase-MB and troponin measurements in acute coronary syndromes. J. Am. Coll. Cardiol. 47(2), 312-318 (2006).

38 Larca LJ, Coppola JT, Honig S. Creatine kinase MB isoenzyme in dermatomyositis: a noncardiac source. Ann. Intern. Med. 94(3), 341-343 (1981).

39 Wolf PL. Abnormalities in serum enzymes in skeletal muscle diseases. Am. J. Clin. Pathol. 95(3), 293-296 (1991).

40 Patel MR, Chen AY, Peterson ED et al. Prevalence, predictors, and outcomes of patients with non-ST-segment elevation myocardial infarction and insignificant coronary artery disease: results from the Can Rapid risk stratification of Unstable angina patients Suppress ADverse outcomes with Early implementation of the ACC/AHA Guidelines (CRUSADE) initiative. Am. Heart J. 152(4), 641-647 (2006).

41 Santopinto JJ, Fox KA, Goldberg RJ et al. Creatinine clearance and adverse hospital outcomes in patients with acute coronary syndromes: findings from the global registry of acute coronary events (GRACE). Heart Br. Card. Soc. 89(9), 1003-1008 (2003).

42 Collinson P, Hammerer-Lercher A, Suvisaari J et al. How well do laboratories adhere to recommended clinical guidelines for the management of myocardial infarction: the CARdiac MArker Guidelines Uptake in Europe Study (CARMAGUE). Clin. Chem. 62(9), 1264-1271 (2016).

-• CARMAGUE study assessing European and North American adherence and differences of guidelines for cardiac biomarkers.

43 Yoshimoto K, Tanaka T, Somiya $\mathrm{K}$ et al. Human heart-type cytoplasmic fatty acid-binding protein as an indicator of acute myocardial infarction. Heart Vessels 10(6), 304-309 (1995).

44 Seino Y, Ogata K, Takano $\mathrm{T}$ et al. Use of a whole blood rapid panel test for heart-type fatty acid-binding protein in patients with acute chest pain: comparison with rapid troponin T and myoglobin tests. Am. J. Med. 115(3), 185-190 (2003).

45 O’Donoghue M, de Lemos JA, Morrow DA et al. Prognostic utility of heart-type fatty acid binding protein in patients with acute coronary syndromes. Circulation 114(6), 550-557 (2006).

46 Tanaka T, Hirota Y, Sohmiya K, Nishimura S, Kawamura K. Serum and urinary human heart fatty acid-binding protein in acute myocardial infarction. Clin. Biochem. 24(2), 195-201 (1991).

47 Loria V, Dato I, Graziani F, Biasucci LM. Myeloperoxidase: a new biomarker of inflammation in ischemic heart disease and acute coronary syndromes. Mediators Inflamm. 2008 (2008). www.ncbi.nlm.nih.gov/pmc/articles/PMC2276594/ 
48 Mullane KM, Kraemer R, Smith B. Myeloperoxidase activity as a quantitative assessment of neutrophil infiltration into ischemic myocardium. J. Pharmacol. Methods 14(3), 157-167 (1985).

49 Zhang R, Brennan ML, Fu X et al. Association between myeloperoxidase levels and risk of coronary artery disease. JAMA 286(17), 2136-2142 (2001).

50 Baldus S, Heeschen C, Meinertz T et al. Myeloperoxidase serum levels predict risk in patients with acute coronary syndromes. Circulation 108(12), 1440-1445 (2003).

- Study by CAPTURE investigators examining the potential usefulness in risk stratification of acute coronary syndrome.

51 Mocatta TJ, Pilbrow AP, Cameron VA et al. Plasma concentrations of myeloperoxidase predict mortality after myocardial infarction. J. Am. Coll. Cardiol. 49(20), 1993-2000 (2007).

- Investigation on myeloperoxidase's long-term outcome in patients after acute MI. 
